\title{
Multimodal Differentiation of Obstacles in Repeated Adaptive Human-Computer Interactions
}

\author{
Felix Putze \\ felix.putze@uni-bremen.de \\ University of Bremen \\ Bremen
}

\author{
Mazen Salous \\ salous@uni-bremen.de \\ University of Bremen \\ Bremen
}

\begin{abstract}
Human Computer Interaction can be impeded by various interaction obstacles, impacting a user's perception or cognition. In this work, we detect and discriminate such interaction obstacles from different data modalities to compensate for them through User Interface (UI) adaptation. For example, we detect memory-based obstacles from brain activity and compensate through repetition of information in the UI; we detect visual obstacles from user behavior and compensate by complementing visual with auditory information in the UI. Online cognitive adaptive systems should be able to decide the most suitable UI adaptation given inputs from several obstacles detectors. In this paper, we employ a Bayesian fusion approach upon different underlying obstacles detectors over multiple consecutive interaction sessions. Experimental results show that the model promisingly outperforms the baseline in the first interaction with an average accuracy of $72.5 \%$ and further improves drastically in subsequent interactions with additional information, with an average accuracy of $98 \%$.
\end{abstract}

\section{CCS CONCEPTS}

- Human-centered computing $\rightarrow$ User models.

\section{KEYWORDS}

Interaction obstacles, multimodal detection, Bayesian fusion, adaptive system

\section{ACM Reference Format:}

Felix Putze and Mazen Salous. 2021. Multimodal Differentiation of Obstacles in Repeated Adaptive Human-Computer Interactions. In 26th International Conference on Intelligent User Interfaces (IUI '21), April 14-17, 2021, College Station, TX, USA. ACM, New York, NY, USA, 10 pages. https://doi.org/10. $1145 / 3397481.3450641$

\section{INTRODUCTION}

Computer systems are used ubiquitously in everyday activity by a variety of users. To improve the usability of such systems for different types of users, customization and adaptation techniques have evolved. While customization is done by the user in dynamic systems which enable users to customize the User Interface (UI)

Permission to make digital or hard copies of all or part of this work for personal or classroom use is granted without fee provided that copies are not made or distributed for profit or commercial advantage and that copies bear this notice and the full citation on the first page. Copyrights for components of this work owned by others than the author(s) must be honored. Abstracting with credit is permitted. To copy otherwise, or republish, to post on servers or to redistribute to lists, requires prior specific permission and/or a fee. Request permissions from permissions@acm.org.

IUI '21, April 14-17, 2021, College Station, TX, USA

(c) 2021 Copyright held by the owner/author(s). Publication rights licensed to ACM ACM ISBN 978-1-4503-8017-1/21/04 . \$ \$15.00

https://doi.org/10.1145/3397481.3450641 according to their specific needs, adaptation is done, in contrast, by the computer system itself. Such adaptive systems estimate the user's states or traits and adapt the UI accordingly in an automatic fashion [30]. Adaptation can be achieved for a larger user group (e.g., children); moreover, for better usability, it may also be realized for individual users in specific situations. While user preferences have been widely analyzed and used for such adaptation (e.g., suggestions based on past browsing and purchase history), we believe that human cognitive processes can be analyzed during Human Computer Interaction (HCI) tasks to further improve adaptation by detecting and compensating potential cognitive interaction $o b$ stacles. That is, individual users may suffer from different interaction obstacles while interacting with computer systems, such as memory-based or visual obstacles. Online detection of different interaction obstacles - for an individual user - enables the system to adapt the UI accordingly to tackle the expected impairment in the interaction task performance.

During an interaction task, human cognitive processes may be impaired by different potential interaction obstacles: a memorybased obstacle (e.g., memory load caused by a secondary task) impairs the short-term memory and a visual obstacle (e.g., colour vision deficiency, where about $8 \%$ of men and $0.5 \%$ women in the world have the common form of colour blindness [18]) impairs the perception process of visually presented information. For example, when the mobile user of a tablet is occupied in parallel with memorizing information they receive during a phone call, their working memory capacity is limited in comparison to an uninterrupted situation; the system can no longer rely on the user remembering all presented information and if this situation goes unnoticed, the interaction will suffer (e.g., when the user forgets which files in a folder they already processed).

Different modalities can be recorded during an interaction task to analyze those impaired cognitive processes and thus to detect the presence of such interaction obstacles and adapt the UI accordingly. For example, we can process the user's behavior captured through interaction with the system or we can record the user's brain activity (e.g., through Electroencephalography, EEG) to capture information about cognitive processes. Generally speaking, different interaction obstacles in an interaction task can be best detected from different modalities using obstacle-specific machine learning models. Thus, different UI adaptations can be applied for different detected interaction obstacles. This is important, because compensating the UI with wrong or unnecessary adaptations can be harmful with regard to interaction task performance and user acceptance. Thus, while online detection of different obstacles can be achieved by different models, an overarching decision model is required to improve the UI with the best adaptation. Because users 
interact with systems not only once, but often several times in succession, such a model must be able to consolidate its result across multiple subsequent interactions and to automatically recover from recognition errors, which may lead to sub-optimal adaptation. Key challenges in creating such a system for detecting interaction obstacles is a) the integration of cues from different sources cues over time, b) to cope with changes or uncertainty, and c) the ability to handle different obstacles which require different adaptation.

In previous work, Salous et al. [25] showed that multiple interaction obstacle can be detected and differentiated from behavioral data through the use of recurrent neural networks. The main contributions of this paper are: 1) Utilization and fusion of multiple complementary modalities to detect and differentiate HCI obstacles more robustly and in parallel, 2) Bayesian approach for such fusion and decision enables consecutive suitability and stability checks until applying the most suitable (best) UI adaptation, and 3) an investigation of the effects of different adaptation mechanisms for different interaction obstacles.

After this introduction, the next sections are structured as follows: in the next section, we discuss related works. After that, we explain the task and the data collection. Then, we present our probability-based model which combines - over consecutive interactions - elementary obstacle detectors models. After that, we evaluate the probability-based model for classification performance over consecutive interactions. Finally, we discuss the probabilitybased results together with questionnaire and game logs analysis results to show that suitable adaptation helps.

\section{RELATED WORK}

In this section, we discuss related works in terms of increasing the usability of computer systems. Concretely, we discuss both customization and adaptation techniques used to tackle the visual and memory-based obstacles discussed in this paper.

In general, the HCI community aims at improving systems usability and user acceptance. Two early works [27], [7] discussed difficulties in integrating Software Engineering (SE) and Human Computer Interaction (HCI) principles and best practices. While both highlighted the importance and difficulty of designing a usabilityconformed UI during the SE life cycle, the authors of [7] also discussed integration proposals between SE and HCI best practices to benefit from $\mathrm{HCI}$ principles while developing computer systems. However, the discussed proposals belong to a static software, i.e., without UI adaptation.

Customization grants users control to customize the UI according to their specific needs. For example, regarding the visual obstacle discussed in this paper, there exist works which introduced a customized UI for potential users with color vision deficiency, see [11] and [23]. According to Jefferson et al. [11], users can customize the colours of any region of the UI. However, there is no detection of the visual obstacle (color vision deficiency) in their interface. An early work for adaptive interfaces is presented by [6], who present an approach to integrate automatic adaptation of a model-driven interface design framework, based on certain user characteristics. Other researchers, like Motti et al. [16] and Minon et al. [14] formalize conceptual frameworks that allow designers to create adaptation rules based on context criteria. An example of an adaptive system for users impeded by visual interaction obstacles is presented by Qaiser et al. [23], who discussed the detection of such an obstacle by utilizing Ishihara plates test (typical color vision test automated in a mobile app). After that test, the most appropriate color scheme for that specific user is chosen. The limitation of such a UI that it is a specific color test cannot be smoothly integrated in HCI applications. This limitation can be tackled by detecting such an obstacle from user behaviour [25]. The authors of [25] introduced also a UI adaptation by utilizing voice instructions to compensate visual obstacles. Sarcar et al. [26] introduce the concept of "ability-based optimization", where they use a cognitive model for evaluation of system variants in an optimization process to account for users with sensorimotor and cognitive impairments.

Adaptation, in contrast to customization, grants control to the adaptive system itself which identifies users and adapts the UI accordingly. For example, regarding the memory-based obstacle discussed in this paper, Sguerra and Jouvelot [28] investigated an adaptation method based on real-time tracking of human working memory. Sguerra et al. modeled the human memory based on the Moran process [15] by maintaining "quanta" numbers (weights) for each stored item. As an HCI task, they used a matching pairs game, tracked the user performance and released an adaptation signal when the performance deteriorates to a value less than a given application-based parameter (no explicit external secondary task obstacle). Thus, the approach by Sguerra and Jouvelot is an explicit performance-based tracking model, which does not consider potential temporal dependencies in behavioral data (user actions) to detect behavioural changes when applying that UI adaptation while an explicit secondary task is present. In contrast, Salous et al. [25] introduced a sequential model, based on Recurrent Neural Networks, that shows advantages in detecting behaviour changes, especially in the presence of memory-based obstacle with UI adaptation. As a dependable adaptive system, Gajos et al. introduced the SUPPLE [8] as an optimization-based automatic adaptive system. It optimizes the UI adaptation task according to the device's constraints (e.g., cell phones, touch panels, etc.). It aims at minimizing the estimated effort for the user's expected actions within an HCI task. While this became a widely used approach, it only targets user experience according to different devices but it does not consider human cognitive processes during HCI tasks.

Several modalities have been applied to detect individual interaction obstacles. Brain activity has often been used to identify interaction obstacles, especially those related to cognitive states of the user. Heger et al. [10] showed how EEG can be classified to detect different levels of cognitive workload, to adapt the interaction behavior of a spoken information system to reduce memory load when the standard behavior would be overwhelming. Dey et al. [3] use EEG to determine a user's task load from the alpha band power in a Virtual Reality (VR) training scenario to adapt the task complexity. They could show that the EEG responded to increases in task load that were not detectable from purely behavioral data. Si-Mohammed et al. [29] detect and differentiate another class of interaction obstacles from EEG, namely visualization errors in VR, such as tracking errors or background anomalies. They show that by exploiting so called error potentials, tracking errors can be detected robustly, while other types of errors could not be identified. A lot of research has been done on the fusion of different 
modalities. D'mello and Kory[5] have shown in a meta-analysis across 90 papers that fusion often helps to improve classification accuracy. Putze et al. [21] demonstrated that the fusion of multiple modalities (EEG and fNIRS) allowed a better finer differentiation of cognitive processes than the use of a single modality. Several techniques can be used for modality fusion. Poria et al. [20] give an overview of methods, ranging from feature-level fusion and linearly weighted late fusion to Bayesian Fusion and other model-based fusion schemes. While recent years saw an increase in deep learning based fusion [31], a fusion scheme based on a Bayesian Network allows a modular combination of data-driven optimization with curated hand-crafting of models.

One shortcoming of the state-of-the-art research is that is usually stops at the initial decision and often only covers a single type of obstacle. The fact that every interaction obstacle requires a specific adaptation, together with the observation that all data-driven methods for automatic adaptation can also yield wrong results, means that the detection process needs to continue after an initial adaptation was triggered. However, it is known that the effects of different cognitive processes interact with each other and thus we should also expect an impact of an active UI adaptation on the detection of interaction obstacles.

\section{DATA COLLECTION}

In this section, we describe the data collection process for the training and evaluation of the interaction obstacle detectors as well as to evaluate the effect of obstacles and adaptation mechanisms. We first introduce the used HCI task and the combinations of interaction obstacles and adaptation mechanisms that were explored. Then, we describe the details of the actual experiment to collect the data.

\subsection{Task and Obstacles}

The HCI task that participants operated during the experiment is a game of matching pairs, a classical family game familiar to most participants. In this game, the player must find pairs of cards which show the same picture, always revealing two cards from a board of face-down cards in each game turn. Pairs which are revealed successfully are removed and the goal of this single-player variant is to clear the board with as few turns as possible. We chose to implement the HCI task as a game as these generate a level of intrinsic motivation [9] and set a natural goal for the user. The game of matching pairs is a typical representation of many HCI tasks as it involves multi-step operations, and many different cognitive functions, including spatial and symbolic working memory, decision making, and planning. These cognitive functions appear prominently in many other HCI tasks, such as machine or vehicle operation, decision support systems, or learning tools.

The task was implemented on an Android tablet and operated by touch on the screen. Figure 1 shows the graphical UI of the game. After a touch on a card, it is turned around and becomes visible. When the second card is revealed, both cards remain visible for $300 \mathrm{~ms}$ and are then turned back face-down. During this time, no new cards can be revealed. Card pictures are represented by uniform colors. We made this choice to avoid semantic correlates that make memorization of some cards easier or more difficult than others. The chosen colors where: red, orange, green, turquoise, yellow, blue, and pink. The implementation of the task was based on an open source memory game ${ }^{1}$ which we customized with experimentspecific cards as well as the ability to toggle different interaction obstacles and adaptation mechanisms (see below). This version of the game is available via Open Science Framework at redacted.

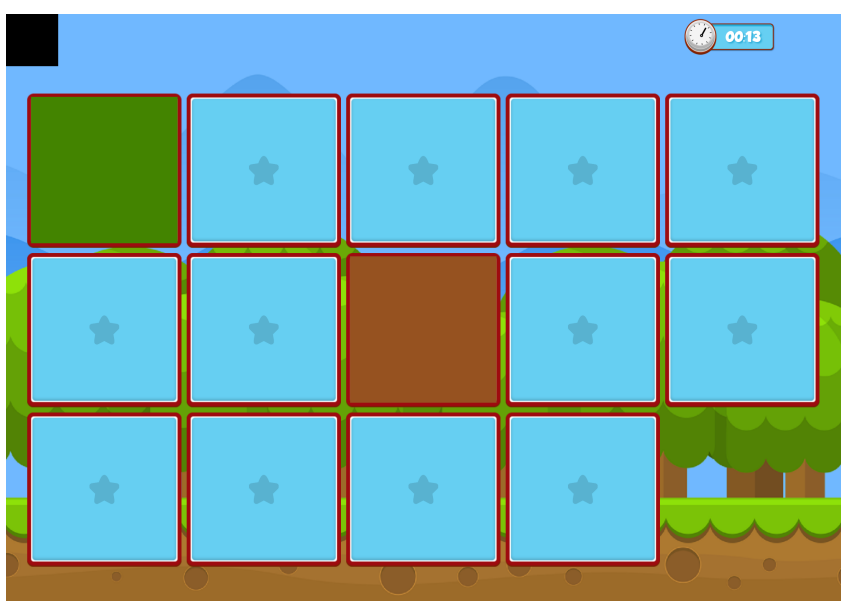

Figure 1: Cards in NoOBs game show well-distinguishable colors [25]

To simulate different interaction obstacles in combination with different types of (appropriate or inappropriate) adaptation, we developed several game modes which are designed to represent such situations. In total, we worked with nine different game modes, which result from the combination of interaction obstacles (including no obstacle) and adaptation (including no adaptation). These combinations include variants of successful adaptation, but also variants in which an adaptation will likely not support the user or even further impede their progress. Such situations can occur during the actual system operation when an initial decision of the system to trigger an adaptation is incorrect. It is important for the system to correct such mistakes pro-actively, to avoid confusion and the necessity of manual intervention. Therefore, we include all combinations in our analysis. Adaptation mechanisms only switch between interaction sessions (which are usually short, with a duration of 1-2 minutes), not within one session.

First, we introduce the three variants for the interaction obstacle:

- No Obstacle (noObs): In this variant, the game is played as described above, without obstacles; the participants were asked to play the game without interaction obstacle, see Figure 1. We expect players to perform best in this variant compared to others with different obstacles, i.e. shortest sequence of rounds is expected to finish this game.

- Memory-based Obstacle (memObs): In this variant, matching pairs is played with a secondary task that induces memory load, to create a volatile memory-based obstacle. Whenever the participant reveals a card, a random number between 1 and 9 is spoken by a synthesized voice. The participant was asked to calculate and memorize the sum of all

${ }^{1}$ https://github.com/sromku/memory-game 


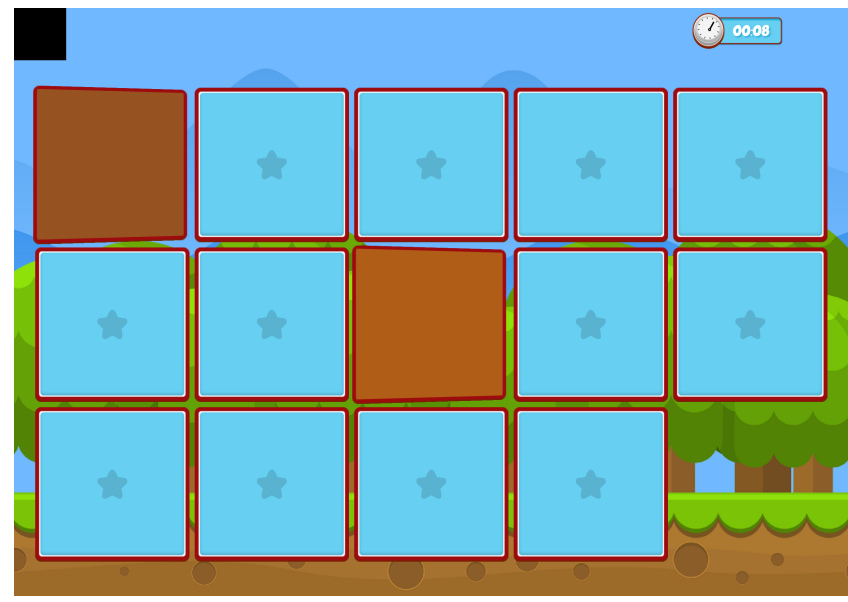

Figure 2: Two (actually different) cards in the VisOBs variant, simulating red-green color vision deficiency

spoken numbers throughout the game. It is well known that mental arithmetic interacts with working memory (WM) [2] and thus such a secondary task limits available WM capacity. This effect works on two different axis: First, the required calculation interferes with the memory encoding of the revealed card; second, the memorized tentative sum is an additional item which needs to be kept in capacity-limited WM. Both mechanisms (distractions and competing items to remember) occur frequently in various $\mathrm{HCI}$ situations, making this task a good representative of a typical interaction obstacle.

- Visual Obstacle (visObs): In this variant, the cards of the game only show different shades of brown color to emulate red-green color vision deficiency as a visual obstacle game variant. The cards are still all different, but are very similar and not easily connected with familiar color labels to memorize card locations. This transformation is done through a brown overlay with $75 \%$ opacity to all cards, moving them closer together in the color space. Figure 2 shows how the affected cards look like in the graphical UI. While the concrete color perception depends on the individual and the exact color-blindness condition, this mode is a good representative of how a color-blind person perceives a user interface with no further (e.g., texture-based) cues to enhance discriminability.

Salous et al. [25] already established that these obstacles indeed influence the objective performance during the HCI task as well as the user's subjective experience.

The three interaction obstacles can be combined with the following three adaptation mechanisms:

- No AdAPtation (NOAdAPT): In this variant, no adaptation is applied, the game is played as determined by the interaction obstacle mode.

- Memory Adaptation (memAdapt): This adaptation mechanism is designed to relieve the player's working memory. For this purpose, we leverage that additional stimulation of past information, especially of that which is not easily remembered through primacy or recency effects [17], leads to rehearsal of information and improves recall probability. Whenever a player reveals non-matching cards, the last two cards prior to these are re-revealed for a very short time. The time of the additional reveal is kept short (225 ms) and skips the usual animations for revealing to avoid extending the time between game turns, which could counter the positive effect of the memory adaptation through additional decay of other information.

- Visual Adaptation (visObs): This adaptation mechanism is tailored towards a support of a user for whom visual perception is impaired. Inspired from theories of multi-resource cognition and perception [32], we do this by leveraging an additional modality: visual information on the playing cards is accompanied by a synthesized voice which represents the card content, utilizing an information channel which is likely not affected by the perceptual obstacle. In our implementation, the letters a,c,j,q,x,v and l were chosen as voice cues, as they are short and easily distinguishable in the German pronunciation. When applied to a situation in which no visual obstacle is present, visual adaptation can have a detrimental effect.

Participants encountered all combinations of interaction obstacles and adaptation mechanisms. When referring to a game mode, we denote it by first naming the obstacle mode and then the applied adaptation. For example, the combination MEMOBS_vIsADAPT denotes the (mismatched) condition in which a visual adaptation is applied to a memory-based interaction obstacle.

\subsection{EEG Recording}

Electrical activity in the human brain shows different characteristics during different brain cognitive processes as well as different types of human behavior; that is, sleeping shows different brain activity compared to walking, memorizing, objects recognition, mental calculations, etc [12]. To record such a brain electrical activity, we use electroencephalography (EEG), which is a noninvasive method, i.e. specific sensors so-called electrodes will be placed along the scalp. Concretely, in our EEG recording setup, we used a BrainProducts actiCHamp recorder with a 32 electrode actiCAP. As recording software, we used the BrainProducts pyCorder. The electrodes were positioned according to the international $10-10$ system. The reference electrode was positioned at the location $\mathrm{Fz}$, while we used these standard ActiCap positions for the other 31 electrodes: Fp1, Fp2, F3, F4, F7, F8, FC1, FC2, FC5, FC6, FT9, FT10, Cz, C3, C4, T7, T8, CP1, CP2, CP5, CP6, TP9, TP10, Pz, P3, P4, P7, P8, Oz, O1, O2. The sampling rate used was $500 \mathrm{~Hz}$, and the impedance was below $10 \mathrm{k} \Omega$. For analyzing the recorded data, we used the frequency domain, i.e. we discriminated EEG signals based on expected different neural oscillations under different conditions, e.g. NOOBS or MEMOBS. We preferred that frequency domain rather than the time domain, because of possible overlapping between multiple stimuli in time domain, e.g. card picture stimulus and spoken number stimulus in case of MEмOвs. EEG data was synchronized with the game events (i.e., revealing a card) through a light sensor of the EEG recorder attached to the tablet above a box switching between black and white triggered by the events. 


\subsection{Study Procedure}

A total of 17 participants was involved in the data collection, with an age between 17 and 27 years and a gender distribution of 13 men and 4 women. Participants were recruited through a local online blackboard. The study was approved by the local ethics committee. All participants gave their explicit written consent for participation in the study. They were compensated with $15 €$.

Participants were instructed to complete each game with as few game turns as possible. Before the actual experiment, every participant had the opportunity to try out the different playing modes in a test game. While playing, participants sat on a chair in front of a desk and were free to either hold the tablet (a Google Pixel C with a 10" screen) in their hand or keep it on the desk. Besides operating the tablet, participants were asked to avoid unnecessary movement to avoid artifacts in the EEG signal. Figure 3 illustrates the recording conditions. After each game, participants were asked for a short self-report of mental demand, playing speed, and ability to memorize the cards on a 7-point Likert scale.

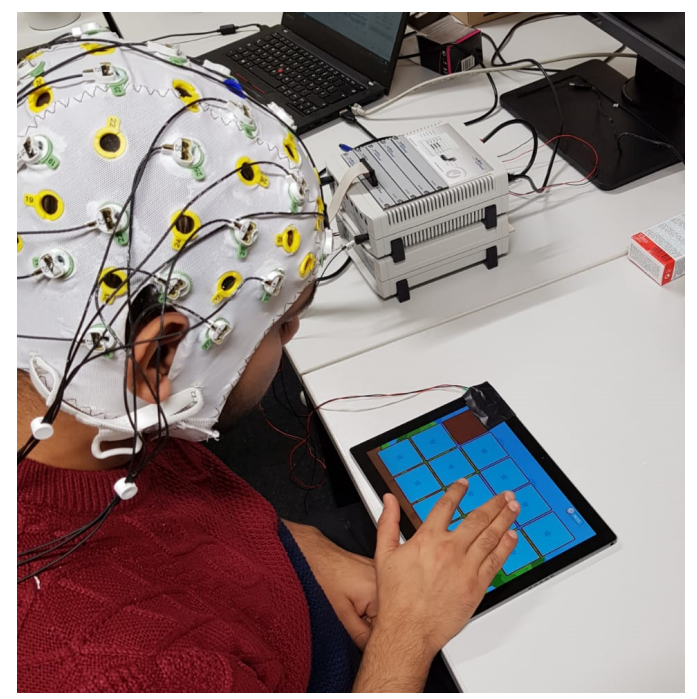

Figure 3: Recording setup of a participant playing the tabletbased matching pairs game while brain activity is recorded through EEG.

In each user study, participants were asked to play multiple game variants in a random order, as described in the section 3.1. In total, every participant played nine games.

\section{INTERACTION OBSTACLE DETECTION}

During the HCI task, we observe neural (EEG) and behavioural data (encoded revealed cards) and pass it to multiple obstacles detectors (in our case, мемOвs and visOвs detectors). Each obstacle detector has the task to detect the presence or absence of one specific obstacle. On top of the individual detectors, we use a Bayesian fusion component, which generates the final decision of choosing an UI adaptation as follows: the outputs of the obstacle detectors (either obstacle or NoOBs) will be passed together with the corresponding confidence scores as inputs to the fusion component, see Figure 5.
The UI adaptation for the next interaction (NOADAPT, MEMADAPT or VISADAPT) is then decided based on the underlying Bayesian model. When the UI adaptation is applied in the next interaction with the same user (i.e., playing a next game round), the same aforementioned steps (observe data, pass it to the detectors, and pass predictions and confidences from detectors to Bayesian fusion model) are repeated to decide on the UI adaptation again and thus either to consolidate the result or to change erroneous UI adaptation. We decided for this architecture (in contrast to a monolithic model discriminating all obstacles at once) as it allows us to flexible add or remove different obstacles and easily consider subsequent multiple interaction.

As a probability-based framework, we use a Dynamic Bayesian Network (DBN) which decides on the most probable obstacle and UI adaptation using inputs from the several underlying detectors over multiple time steps.

\section{MULTIMODAL OBSTACLE DETECTORS}

An obstacle detector is a system component that represents a binary classifier to detect the presence or absence of a specific obstacle in a (segment of an) interaction session from a specific modality.

As users often engage in multiple subsequent HCI sessions, we also must not only regard each such session in isolation, but also in context. This includes the fact that the system may have already triggered an adaptation mechanism based on the result of obstacle detection in earlier sessions. As the initial adaptation decision might have been wrong and as volatile interaction obstacles might disappear if conditions change, it is important to still monitor interaction obstacles even after the first sessions. However, adaptation may influence the recorded data in different ways, usually by mitigating the effect of the obstacle (in case that the adaptation fits the obstacle), but sometimes also by augmenting it (in case the adaptation and the obstacle are mismatched due to a detection error). Therefore, we train multiple instances of each obstacle detector, each one in the presence of one adaptation mode (incl. NOADAPT as the base case).

Each obstacle detector only focuses on one modality and the detection of one obstacle. In contrast to the alternative of training one monolithic classifier which integrates multiple modalities on a feature level and differentiates multiple interaction obstacles at the same time in a multi-class model, this modular approach has the advantage of maximum flexibility: when new modalities become available or have to be removed (e.g., due to a broken sensor) or when a new obstacle needs to be treated, the set of detectors can be adjusted easily.

\subsection{Behavioral Model for Visual Obstacles}

For the classification of the behavioral data, we follow the work of Salous et al. [25], who employ a recurrent neural network (Long Short-Term Memory, LSTM) applied to sequences of user interactions for the classification of interaction obstacles. In the case of the matching pairs game, user actions correspond to revealed cards and these are encoded by their content and identity on the playing board. As the number of game episodes to train an LSTM is limited, we use a simulator to generate additional training episodes. The simulator is based on the Cognitive Memory Model [22] and 


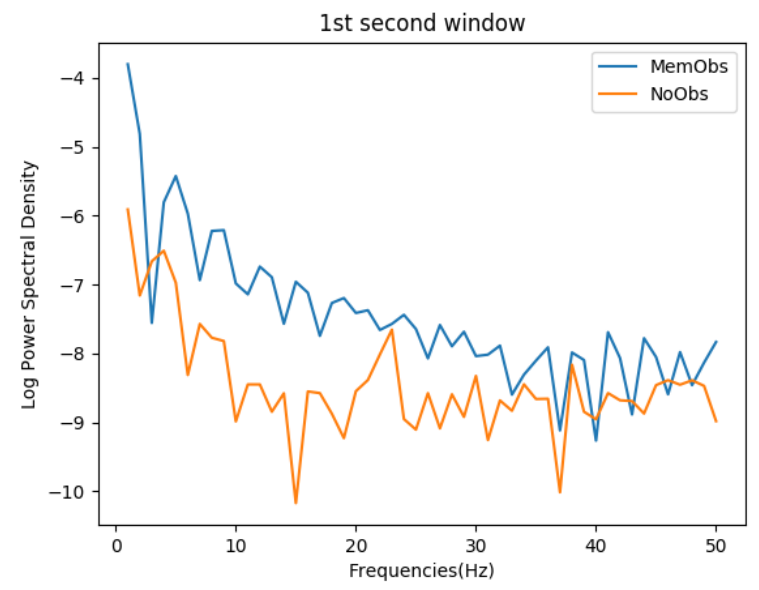

Figure 4: Averaged EEG power spectra observed at electrode Fp1 after revealing a card from a NoOBs game and from МемОвs game.

represents spatial and symbolic working memory, as well as a playing strategy which balances exploration of unknown cards and exploitation of known pairs. See the original work for additional details of the model architecture and training parameters.

The model used here differs from the one reported in [25] in four ways: First, we train models for the different adaptation modes, while [25] only applies models for the NOADAPT condition. Second, we reduce the amount of simulated data used for training. Third, we train multiple models from random initializations and perform a voting among them to increase overall robustness of the classification. Finally, the model yields a confidence estimation based on the probability distribution expressed in the final softmax layer.

\subsection{Brain Activity Model for Memory Obstacles}

For the detection of interaction obstacles from brain activity, we expect EEG signals to be discriminant between naturally different mental tasks, e.g. EEG signals when playing the matching pairs task only (NOOBs) vs. EEG signals when playing matching pairs while being under additional memory load (мемОвs). We can see the expected difference in spectral power for the two conditions in Figure 4.

For classification, we extracted episodes of $1000 \mathrm{~ms}$ (using a Hanning window) following the reveal event of each card after clicking it. We chose this segment as it can be connected with a relatively clear cognitive process of recall and memorization after a new card has been revealed, reducing the variance compared to a sliding window across different segments of the data. Data from each channel is normalized to zero mean to account for offsets and slow drifts.

To extract features for classification, we follow Kumar et al. [12], who reviewed several EEG-based prediction tasks and summarized results as which EEG band is recommended to which task. According to their review results, the power in the Delta band $(0-4 \mathrm{~Hz})$ increases during complex mental tasks (arithmetic tasks). Additionally, the review results show that higher working memory loads have been reported to cause increase in low beta bands $(12-18 \mathrm{~Hz})$ powers in frontal mid-line regions. Thus, after processing the EEG signals and preparing them in the frequency domain using Fast Fourier Transformation, we filter the resultant EEG frequencies (in bins of width $1 \mathrm{~Hz}$ ) regarding to a combination of both delta and low beta bands. Consequently, the classifier is passed on 10 features per revealed card. We stacked the features for all electrodes (except the reference at Fz) in one feature vector with 310 (10 features each for 31 electrodes) features passed to the model per revealed card.

For the choice of classifier, we follow Lotte [13], who finds Support Vector Machines (SVM) to be effective for the classification of EEG signals. They also report that for typical small EEG data sets, neural networks rarely show performance improvements compared to other methods. We use an SVM model with default parameters (Radial Basis Function (RBF) kernel, $C=1.0, \gamma=0.1$ ) to classify EEG data into its classes ${ }^{2}$, NоOBs on the one hand and мЕмОвs on the other hand. The classifier is trained in a person-independent fashion, using z-normalized features of all training participants. For the integration of information over time, we aggregate the SVM predictions of the first 10 turns (20 cards) and vote the predicted label and average the corresponding confidences of the individual SVM predictions for the whole sequence. More concretely, we assign the label with the highest average confidence calculated according to Platt scaling [19]. We perform this aggregation for two reasons: 1) If the event-based classification performs better than the random baseline, such a confidence-based aggregation is expected to improve the prediction accuracy, and 2) it enables a comparison to the behavioral predictions which are based on sequences.

\section{BAYESIAN MODELING OF SUBSEQUENT ADAPTIVE SESSIONS}

Behavioral and neural data now give two complementary perspectives on the presence or absence of interaction obstacles. Naturally, we are interested in the combination of both modalities; however, one challenge is the very different nature of both models, using different machine learning algorithms and different time scales and sampling rates. Also, each interaction obstacle detector is only trained to detect the presence of absence of one interaction obstacle and is not designed to differentiate between multiple obstacles. To tackle these challenge, we employ a Bayesian late fusion scheme which takes the classification result and a confidence estimate of each individual interaction obstacle detector as input. From the output of the Bayesian model, we derive the decision for the adaptation mechanism which is applied in the next interaction session. Beyond the fusion of modalities for a single session, the task of the Bayesian model is also the integration over multiple interaction sessions. This is accomplished through the use of a dynamic Bayesian model, which takes inputs from previous time steps, including the adaptation decision. The underlying model architecture is shown in Figure 5.

For implementation, we use the Bayesian Network (BN) in PGMPY [1] to create an unrolled Dynamic Bayesian Network (DBN) which

\footnotetext{
${ }^{2}$ we decided not to optimize SVM parameters on hold-out data to avoid overfitting on the relatively small sample size.
} 
learns the underlying Conditional Probabilistic Distributions (CPDs) directly from data in consecutive interactions of the same user. In unrolling, the UI adaptation decision in the first interaction is fed to the UI adaptation node in the subsequent interaction to benefit from such an information when re-deciding the UI adaptation (see the self loop in UI Adaptation node in the Figure 5).

Note that the chosen adaptation in a follow-up interaction is known as it was chosen by the system in a previous iteration. Therefore, we can always chose the appropriate obstacle detectors trained on data of the corresponding adaptation mode. For example: if the UI adaptation decided in the first interaction for MEMADAPT, then the DBN chooses the following models for the next interaction: MEMOBS detector given MEMADAPT (NOOBS_MEMADAPT vs. MEMOBS_MEMADAPT) and VISOBs detector given MEMADAPT (NOOBS_MEMADAPT vs. visOBS_MEMADAPT). We also point out that while we only maintain an EEG-based model for the detection of MEMOBS and a behavior-based detector of visOBs, the DBN always regards both modalities jointly as it decides between adaptations for both obstacles at the same time.

The introduced design in Figure 5 allows a flexible addition of more models for new different obstacles. That is, similar to the EEG-based мЕмОвs detector and behaviour-based visOBs detector, new detectors for new obstacles can be trained and added to the overall cognitive adaptive model depicted in Figure 5.

\section{EVALUATION}

In this section, we evaluate the proposed model for the detection of interaction obstacles and selection. For this purpose, we first evaluate the obstacle detectors individually for all nine combinations of interaction obstacles and adaptation mechanisms. This is followed by an evaluation of the final DBN result to investigate the detection performance of the fusion as well as the effect of temporal integration. Finally, we look at the impact of adaptation mechanisms on the performance in the presence of different interaction obstacles, to study the importance of correct differentiation.

\subsection{Evaluation of Obstacle Detectors}

As classes are balanced between all combinations of interaction obstacles and adaptation mechanisms, we can use classification accuracy as performance metric. The baseline for all the obstacle detectors is $50 \%$ (binary classification: NoOBs vs. OBSTACLE). We apply a leave-one-subject-out strategy as a cross-validation method to train and test both SVM and LSTM. For each fold, given the dataset recorded from the 17 participants, the SVM is trained from the EEG data of 16 participants and tested for the EEG data from the remaining participant, i.e. both detectors are trained and evaluated as person-independent models. Similarly, the LSTM is trained, for each fold, from the real behavioural data and the corresponding simulated behavioral data from 16 participants and tested for the remaining participant. To validate our decision to use the EEG-based detector for мЕмОвs and the behavior-based detector for visOвS, we also train the detectors in the respective opposite condition.

Figure 6 shows confusion matrices for all the obstacle detectors applied to data under all obstacles and adaptation mechanisms. The columns indicate the detectors according to target obstacle and modality, e.g., behaviour-based мемOвs. The rows indicate the UI adaptation which was present: NOADAPT, MEMADAPT and VISADAPT. We see that on average, the proposed combinations (EEG-based detector for мЕмОвs and the behavior-based detector for visOBs) outperform the other variants, validating that choice. The EEG-based мЕмOвs detector achieves an accuracy of $82.4 \%$ for both the NOADAPT and VISADAPT condition. The weaker result of $61.7 \%$ for the MEMADAPT condition is plausible as that adaptation is designed to attenuate the effect of the memory obstacle, making the differentiation more difficult. The behavior-based detector does not respond in this fashion and is weaker in every condition. We see similar patterns for visOBs detection (third and fourth column), only that in this case the behavior-based model outperforms the EEG-based detector on average.

\subsection{DBN Evaluation Through Consecutive Interactions}

We evaluate the DBN according to its ability of deciding the correct UI adaptation: NOADAPT, MEMADAPT or VISADAPT, thus, the baseline accuracy is $33.33 \%$. We evaluate the DBN for consecutive interactions; that is, after the first interaction is finished (random matching pairs game variant: NOOBS, МЕMOBS or vISOBS), a UI adaptation is made by the DBN (NOADAPT, MEMADAPT or VISADAPT) and can be evaluated. Similarly, after that UI adaptation decision is applied, subsequent interactions can be supported by the DBN with a UI Adaptation decision (either to keep the same UI adaptation or to change). Those decisions for the subsequent interactions can be also evaluated to show how well does the DBN learn from more information available through consecutive interactions.

For this purpose, we generate sequences of interaction sessions of the same person with constant interaction obstacle, but potentially changing adaptation mode. This simulates the system applying the adaptation modes considered optimal based on the data of one session, while maintaining the ability to re-evaluate the decision once additional data becomes available.

Figure 7 shows that the DBN promisingly outperforms the baseline in the first interaction with $72.5 \%$ as an average accuracy ${ }^{3}$. Furthermore, it shows that the DBN further learns through the next interaction and improves the average accuracy to $98 \%$. For more concrete evaluation, we look at confusion matrices for first and second interactions, moreover, in the second interaction, we look also at a confusion matrix for both correct and wrong decisions from the first interaction, see Figure 7. The confusion matrices of first and second interaction in Figure 7 show that the prediction accuracy of all labels improves when re-deciding the UI adaptation in the second interaction. That is, the accuracy of all the predicted labels in the first interaction improves from $82.4 \%, 82.4 \%$, and $52.9 \%$ to $100 \%, 96 \%$, and $98 \%$ for NOADAPT, MEMADAPT and VISADAPT respectively. We observe the greatest improvement for the VISADAPT case (a relative improvement of $84.9 \%$ ) as the model is able to recover many cases in which the original visOBs was not detected. This is possible because the the VISADAPT class does not exhibit false positives in the first interaction and evidence can accumulate. Indeed, the rightmost part of Figure 7 shows that in $100 \%$ of all

\footnotetext{
${ }^{3}$ note that the confusion matrices are now labeled with the adaptation modes, compared to the labeling with interaction obstacles which is done for the obstacle detectors, as the adaptation mode is the output of the DBN.
} 


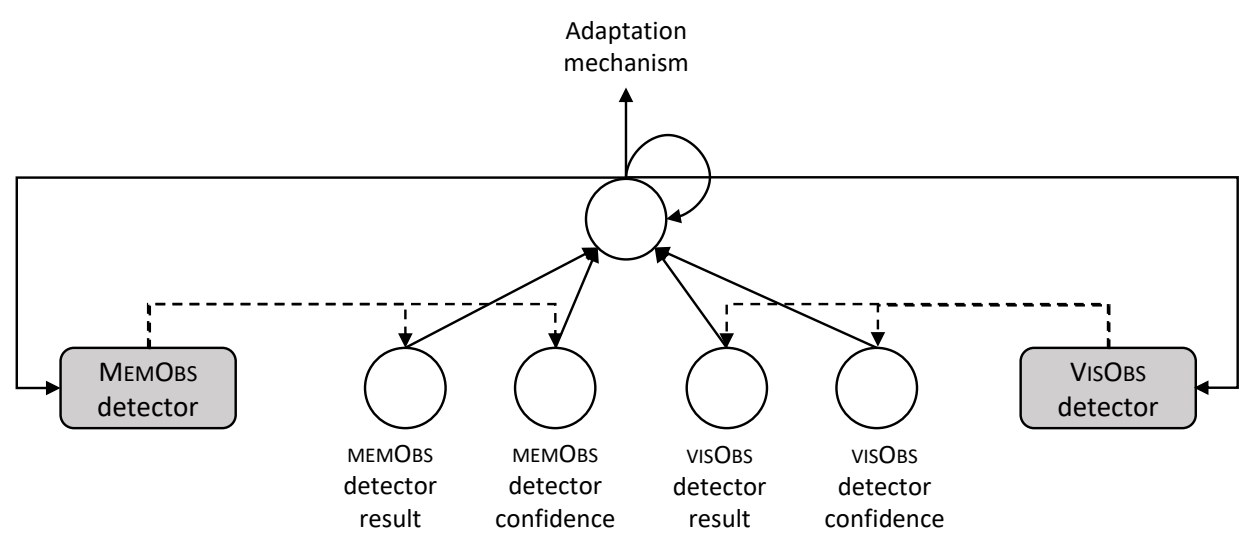

Figure 5: Architecture of the Dynamic Bayesian Network for the derivation of adaptation mechanisms suited for the detected interaction obstacle.
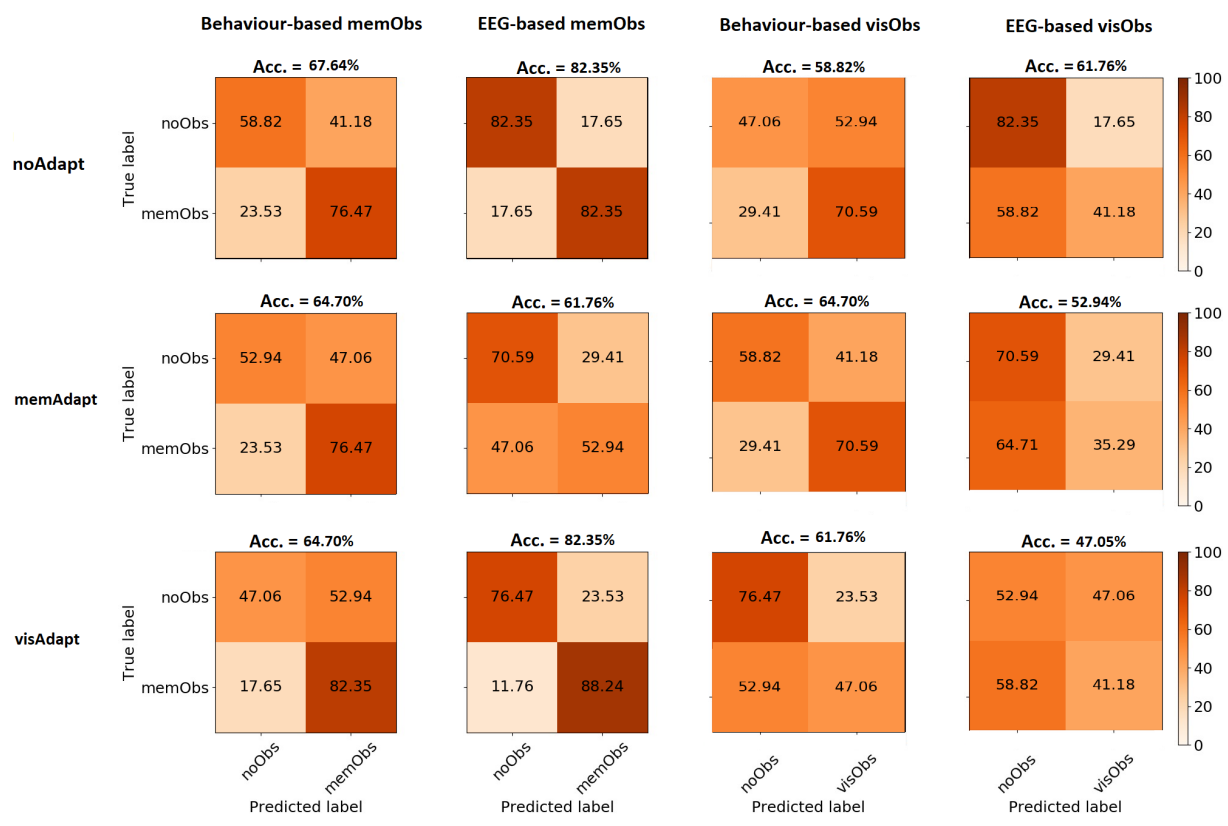

Figure 6: Elementary models evaluation. Upper matrices: noAdapt games (first interaction). Middle matrices: memAdapt games (second interaction). Lowest matrices: VisAdapt games (second interaction).

cases could wrong decisions in the first interaction be corrected in the second one. Note that not all combinations appear in the evaluation, for example there are no confusions between MEMADAPT and VISADAPT in the first interaction and thus, the corresponding combinations are not evaluated in the second iteration. Still, these cases were trained in the model and the results in Section 7.1 make it likely that detection performance will be on similar levels.

\subsection{Evaluation of Adaptive Strategies}

Finally, we look at the impact of different adaptation modes on the usability during a session. The main purpose of the detection and discrimination of several interaction obstacles is that we expect different adaptations to be optimal for different types of obstacles, i.e., there is no "silver bullet" adaptation (which could be activated by default). To investigate the validity of this claim and the effectiveness of the adaptation, we perform a two-way ANOVA with the obstacle mode and adaptation mode as independent variables and the self-reported mental demand as dependent variable.

We see a significant main effects of both obstacle mode (as expected, because especially the memory obstacle has a strong impact on the mental mental demand) with $F=131.49, p=2.7 e^{-21}$, and $\eta=0.47$ as well as for adaptation mode (which shows that adaptation is effective) with $F=2.67, p=0.007$, and $\eta=0.02$. Besides, we observe an interaction effect $\left(F=7.67, p=7.2 e^{-4}\right.$, and $\left.\eta=0.05\right)$, 

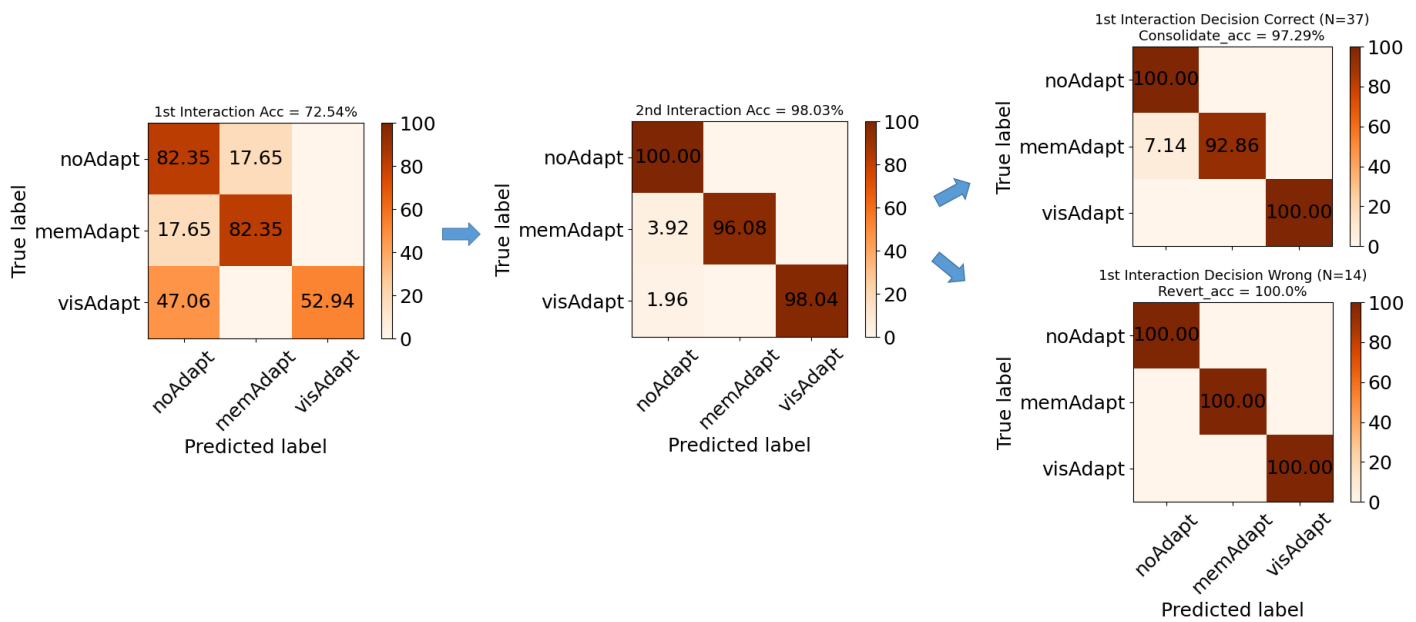

Figure 7: Left: DBN first interaction evaluation. Middle: DBN second interaction evaluation overall decisions from $1^{\text {st }}$ interaction. Right: detailed evaluation for the second interaction given correct (upper matrix) and wrong (lower matrix) decisions from the first interaction.

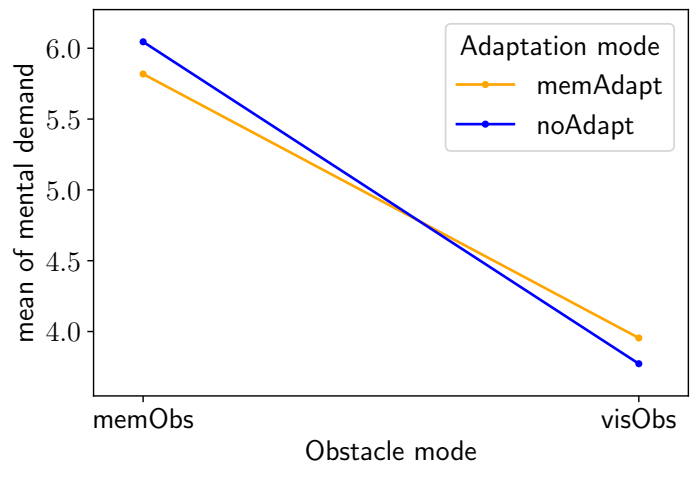

(a) MEMADAPT

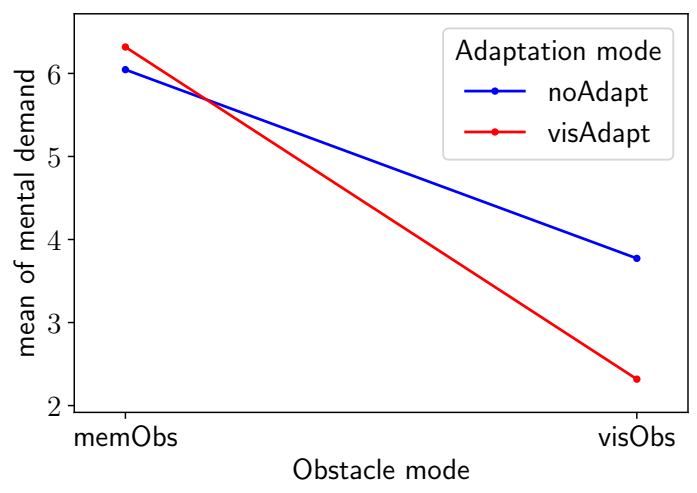

(b) VISADAPT

Figure 8: Interaction between adaptation mechanisms and interaction obstacles for experienced mental demand. which shows that indeed MEMADAPT is helpful to support the user in case of а мемОвs (i.e., it reduces mental load), but is detrimental otherwise. The same holds for visAdAPT. Figure 8 illustrates the interaction effect. We observe similar effects for other usability metrics, such as playing speed, number of revealed cards, or self-assessment of playing performance.

\section{DISCUSSION \& CONCLUSION}

The results show that by combining behavior-based and EEG-based obstacle detectors, we are able to differentiate between multiple interaction obstacles, even in the presence of different active adaptation modes. This enables the use of information across multiple interaction sessions to validate or correct an initial adaptation decision. This drastically improves classification accuracy; in a second session, the system was able to predict the prevalent interaction obstacle (if any) with near-perfect accuracy. This allows the system to either confirm or correct any adaptation mechanisms triggered after the initial session. While we performed the presented analysis in an offline batch mode, they were done through a real-time capable system that can switch.

It remains to discuss the implications of these results on general HCI systems. While we investigated the detection of interaction obstacles in a specific setting, the chosen memory game seems to be a good fit to represent many typical HCI tasks: It involves visual inspection of the UI, working memory retrieval and encoding (of both spatial and symbolic information), as well as planning and decision making, which all occur also in many other HCI tasks. Similarly, the investigated interaction obstacles touch generic impediments to perception and cognition encountered in many different contexts. Therefore, the employed models are relatively generic and allow a transfer to different tasks or conditions: The EEG-based мЕмОвs detector depends on a trigger for specific system events to segment windows, but this can be easily obtained from most applications. The behavior-based visOBs detector relies more on 
specific characteristics of the task and will need to be retrained for a different target application; however as it is based on logged user interaction, the necessary training data is easy to acquire in the background of a newly enrolled application.

One limitation of the presented work is that the interaction obstacles were only simulated for the purpose of a controllable study with comparable conditions between all participants. Previous work [24] has shown that the described approach also works with real interaction obstacles, namely naturally limited working memory capacity. The investigated obstacles were chosen to cover both persistent and volatile obstacles as well as obstacles resulting from impairment of perception as well as internal cognition. Nevertheless, future work needs to further tackle the challenge of transferring the developed models to users experiencing real interaction obstacles. This also involves the EEG recording; while no movement restraints were in place for the participants in the current study, a data collection in real life conditions will still add more and different artifacts (e.g., caused by distracting events, extended physical activity, etc.). To achieve the current results, no extended EEG artifact removal was necessary; this makes us optimistic that there is enough untapped potential to handle data from situations of higher ecological validity, as has been discussed for example by Ding et al. [4]. Finally, due to COVID-19 related contact restrictions, we unfortunately could not yet perform a usability evaluation of the whole system, although the real-time capable end-to-end system is available. This will be made up for as soon as the situation allows it again.

\section{ACKNOWLEDGMENTS}

This research was supported by the DFG, under grant no. 316930318, "Detection of Interaction Competencies and Obstacles".

\section{REFERENCES}

[1] Ankur Ankan and Abinash Panda. 2015. pgmpy: Probabilistic graphical models using python. In Proceedings of the 14th Python in Science Conference (SCIPY 2015) SciPy Organizers, Austin, USA, 6-11.

[2] Edward H Chen and Drew H Bailey. 2020. Dual-task studies of working memory and arithmetic performance: A meta-analysis. fournal of Experimental Psychology: Learning, Memory, and Cognition 47(2) (2020), 220-233.

[3] Arindam Dey, Alex Chatourn, and Mark Billinghurst. 2019. Exploration of an EEG-based cognitively adaptive training system in virtual reality. In 2019 IEEE Conference on Virtual Reality and 3D User Interfaces (VR). IEEE, Osaka, Japan, $220-226$.

[4] Yi Ding, Brandon Huynh, Aiwen Xu, Tom Bullock, Hubert Cecotti, Matthew Turk, Barry Giesbrecht, and Tobias Höllerer. 2019. Multimodal Classification of EEG During Physical Activity. In 2019 International Conference on Multimodal Interaction. ACM, Suzhou, Jiangsu, China, 185-194.

[5] Sidney K D'mello and Jacqueline Kory. 2015. A review and meta-analysis of multimodal affect detection systems. ACM Computing Surveys (CSUR) 47, 3 (2015), $1-36$.

[6] Jacob Eisenstein and Angel Puerta. 2000. Adaptation in automated user-interface design. In Proceedings of the 5th international conference on Intelligent user interfaces. ACM, New Orleans, USA, 74-81.

[7] Xavier Ferre, Natalia Juristo, and Ana M Moreno. 2006. Obstacles for the integration of hci practices into software engineering development processes. In Encyclopedia of Human Computer Interaction. IGI Global, Hershey, USA, 422-428.

[8] Krzysztof Gajos and Daniel S Weld. 2004. SUPPLE: automatically generating user interfaces. In Proceedings of the 9th international conference on Intelligent user interfaces. ACM, Funchal, Portugal, 93-100.

[9] Eduardo H Calvillo Gámez, Paul Cairns, and Anna L Cox. 2009. From the gaming experience to the wider user experience. In People and Computers XXIII Celebrating People and Technology. Swindon, Cambridge, UK, 520-523.

[10] Dominic Heger, Felix Putze, and Tanja Schultz. 2011. An EEG Adaptive Information System for an Empathic Robot. International fournal of Social Robotics 3, 4 (2011), 415-425. https://doi.org/10.1007/s12369-011-0107-x
[11] Luke Jefferson and Richard Harvey. 2007. An interface to support color blind computer users. In Proceedings of the SIGCHI conference on Human factors in computing systems. ACM, San Jose, USA, 1535-1538.

[12] Jyotish Kumar et al. 2016. Affective modelling of users in HCI using EEG. Procedia Computer Science 84 (2016), 107-114.

[13] Fabien Lotte. 2014. A tutorial on EEG signal-processing techniques for mentalstate recognition in brain-computer interfaces. In Guide to Brain-Computer Music Interfacing. Springer, Cham, Switzerland, 133-161.

[14] Raúl Miñón, Fabio Paternò, and Myriam Arrue. 2013. An environment for designing and sharing adaptation rules for accessible applications. In 5th SIGCHI symposium on Engineering interactive computing systems. ACM, London, UK, 43-48.

[15] Patrick Alfred Pierce Moran. 1958. Random processes in genetics. In Mathematical proceedings of the cambridge philosophical society, Vol. 54(1). Cambridge University Press, Cambridge, UK, 60-71.

[16] Vivian Genaro Motti and Jean Vanderdonckt. 2013. A computational framework for context-aware adaptation of user interfaces. In 7th International Conference on Research Challenges in Information Science (RCIS). IEEE, Paris, France, 1-12.

[17] Bennet B Murdock Jr. 1962. The serial position effect of free recall. fournal of experimental psychology 64, 5 (1962), 482.

[18] Conrado F Ostia, Dionis A Padilla, Febus Reidj, G Cruz, Roy Jayson D Galang, Anne Shirley M Josafat, and Edrick F Victoria. 2019. Electronic Vision System with Personalized Calibration of Color Compensation for People with Partial Color Vision Deficiency Using Raspberry Pi Digital Image Processing. In 2019 5th International Conference on Control, Automation and Robotics (ICCAR). IEEE, Beijing, China, 315-319.

[19] John Platt et al. 1999. Probabilistic outputs for support vector machines and comparisons to regularized likelihood methods. Advances in large margin classifiers 10, 3 (1999), 61-74.

[20] Soujanya Poria, Erik Cambria, Rajiv Bajpai, and Amir Hussain. 2017. A review of affective computing: From unimodal analysis to multimodal fusion. Information Fusion 37 (2017), 98-125.

[21] Felix Putze, Sebastian Hesslinger, Chun-Yu Tse, YunYing Huang, Christian Herff, Cuntai Guan, and Tanja Schultz. 2014. Hybrid fNIRS-EEG based classification of auditory and visual perception processes. Frontiers in neuroscience 8 (2014), 373.

[22] Felix Putze, Tanja Schultz, Sonja Ehret, Heike Miller-Teynor, and Andreas Kruse. 2015. Model-based Evaluation of Playing Strategies in a Memo Game for Elderly Users. In 2015 IEEE International Conference on Systems, Man, and Cybernetics. IEEE, Hong Kong, China, 929-934.

[23] Abu Zohran Qaiser and Muhammad Taha Khan. 2017. Adaptive Interface for Accommodating Colour-Blind Users by Using Ishihara Test. arXiv:1712.03329 [cs.HC]

[24] Mazen Salous and Felix Putze. 2018. Behaviour-Based Working Memory Capacity Classification Using Recurrent Neural Networks. In 26th European Symposium on Artificial Neural Networks, Computational Intelligence and Machine Learning. ESANN, Bruges, Belgium, 159-164.

[25] Mazen Salous, Felix Putze, Markus Ihrig, and Tanja Schultz. 2019. Visual and Memory-based HCI Obstacles: Behaviour-based Detection and User Interface Adaptations Analysis. In IEEE International Conference on Systems, Man, and Cybernetics (IEEE SMC 2019). IEEE, Bari, Italy, 1664-1671.

[26] Sayan Sarcar, Jussi PP Jokinen, Antti Oulasvirta, Zhenxin Wang, Chaklam Silpasuwanchai, and Xiangshi Ren. 2018. Ability-based optimization of touchscreen interactions. IEEE Pervasive Computing 17, 1 (2018), 15-26.

[27] Ahmed Seffah and Eduard Metzker. 2004. The obstacles and myths of usability and software engineering. Commun. ACM 47, 12 (2004), 71-76.

[28] Bruno Sguerra and Pierre Jouvelot. 2019. An Unscented Hound for Working Memory and the Cognitive Adaptation of User Interfaces. In Proceedings of the 27th ACM Conference on User Modeling, Adaptation and Personalization. ACM, Larnaca, Cyprus, 78-85.

[29] Hakim Si-Mohammed, Catarina Lopes-Dias, Maria Duarte, Ferran Argelaguet, Camille Jeunet, Géry Casiez, Gernot R Müller-Putz, Anatole Lécuyer, and Reinhold Scherer. 2020. Detecting system errors in virtual reality using EEG through error-related potentials. In 2020 IEEE Conference on Virtual Reality and 3D User Interfaces (VR). IEEE, Atlanta, USA, 653-661.

[30] Horst Treiblmaier, Maria Madlberger, Nicolas Knotzer, and Irene Pollach. 2004. Evaluating personalization and customization from an ethical point of view: an empirical study. In 37th Annual Hawaii International Conference on System Sciences, 2004. Proceedings of the. IEEE, Big Island, USA, 10-pp.

[31] Valentin Vielzeuf, Alexis Lechervy, Stéphane Pateux, and Frédéric Jurie. 2018. Centralnet: a multilayer approach for multimodal fusion. In Proceedings of the European Conference on Computer Vision (ECCV) Workshops. Springer, Munich, Germany, 575-589.

[32] Christopher D Wickens. 2008. Multiple resources and mental workload. Human factors 50, 3 (2008), 449-455. 